Stud. Univ. Babeş-Bolyai Math. 65(2020), No. 4, 651-659

DOI: $10.24193 /$ subbmath.2020.4.11

\title{
The size of some vanishing and critical sets
}

\author{
Cornel Pintea
}

\begin{abstract}
We prove that the vanishing sets of all top forms on a non-orientable manifold are at least 1-dimensional in the general case and at most 1-codimensional in the compact case. We apply these facts to show that the critical sets of some differentiable maps are at least 1-dimensional in the general case and at most 1-codimensional when the source manifold is compact.
\end{abstract}

Mathematics Subject Classification (2010): 57R70, 57R35, 57M10.

Keywords: Critical and vanishing sets.

\section{Introduction}

It is well-known that the orientability of a manifold is characterized by the existence of a top differential form which never vanishes. Therefore it is natural to investigate the size of the vanishing sets $V(\theta):=\left\{p \in M: \theta_{p}=0\right\}$ of the top forms $\theta \in \Omega^{m}(M)$ towards a measure of the deviation from orientability of the involved nonorientable manifold $M$. Indeed, the complement of every vanishing set of a top form is orientable and the smallest such vanishing sets are good candidates to measure this deviation. In this paper we show that the top forms of non-orientable manifolds cannot have arbitrarily small vanishing sets and apply this fact to show that some maps cannot have arbitrarily small critical sets. For instance the zero dimensional subsets of the non-orientable manifolds are neither vanishing sets of the top differentiable forms, nor critical sets of any differentiable function with orientable regular set, for the orientable option of the target manifold. Similar lower bounds for the size of the branch locus arise due to Church and Timourian $[5,6]$ in the codimension cases 0 , -1 and -2 . On the other hand, the critical set of a zero codimensional differentiable map was treated before in [17], where the critical set is realised as the vanishing set of the pull-back of a volume form on the oriented target manifold.

Note that the other extreme is well represented in the recent years, as quite some effort oriented towards the maps with finite critical sets has been done, not only for one dimensional, but also for higher dimensional target manifolds $[1,2,3,8,9,10]$. 
The paper is organized as follows: In the second and third sections we quickly review the tools and emphasize the preparatory results needed to prove the main results of the paper, which are also stated here. In the fourth section we prove the main results of the paper, the first of which concerns the surjectivity of the group homomorphism induced, at the level of fundamental groups, by the inclusion $M \backslash A \hookrightarrow$ $M$, where $M^{m}(m \geq 2)$ is a manifold and $A \subset M$ is a closed zero dimensional set. As a consequence we observe that the dimension of the critical set of a zero or lower codimensional map, whose target manifold is orientable and the source manifold is non-orientable, is at least 1-dimensional. Relying, all over this paper, on the inductive definition of the 'dimension' [7, 13], we prove that the dimension of the critical set of a zero or lower codimensional map, whose target manifold is compact orientable and the source manifold $M^{n}$ is compact non-orientable, is at least $(n-1)$-dimensional. Recall however that the small and large inductive dimensions are equal to each other and both are equal with the covering dimension whenever the evaluated space is separable [7, p. 65]. Since differential manifolds are metrizable metric spaces, it follows that the inductive dimensions of a certain subset of a given manifold are equal to each other and both are equal with the covering dimension of that subset.

\section{Main results}

In order to achieve such results we rely on the characterization of orientability of a connected differential manifold $M$ by means of the orientation character, i.e. the group homomorphism $w_{M}: \pi_{1}(M) \longrightarrow C_{2}:=\{-1,1\}$ defined by

$$
w_{M}([\gamma])=\left\{\begin{aligned}
1 & \text { if } \tilde{\gamma}(1)=\tilde{x}_{1} \\
-1 & \text { if } \tilde{\gamma}(1)=\tilde{x}_{-1},
\end{aligned}\right.
$$

where $\tilde{\gamma}:[0,1] \longrightarrow \hat{M}$ is the lift of the loop $\gamma:[0,1] \longrightarrow M, \gamma(0)=\gamma(1)=x$, with $\tilde{\gamma}(0)=\tilde{x}_{1}, p: \hat{M} \longrightarrow M$ is the orientable double cover of $M$ and $p^{-1}(x)=\left\{\tilde{x}_{1}, \tilde{x}_{-1}\right\}$. Indeed, $M$ is orientable if and only if the orientation character is trivial. Equivalently, $M$ is non-orientable if and only if $w_{M}$ is onto. Taking into account that the orientation double cover of $O$ is $\left.p\right|_{p^{-1}(O)}: p^{-1}(O) \longrightarrow O$, we deduce that the orientation character of a connected open set $O \subseteq M$ can be decomposed as

$$
\omega_{O}=w_{M} \circ \pi_{1}\left(i_{O}\right), \text { where } \pi_{1}\left(i_{O}\right): \pi_{1}(O) \longrightarrow \pi_{1}(M)
$$

is the group homomorphism induced by the inclusion map $i_{O}: O \hookrightarrow M$. Consequently the open connected subset $O$ of a non-orientable manifold $M$ remains non-orientable whenever $\pi_{1}\left(i_{O}\right)$ is surjective. Note that the orientation character $\omega_{M}$ of $M$ coincides with $w_{1}(M) \circ \rho$, where $\rho: \pi_{1}(M) \longrightarrow H_{1}(M, \mathbb{Z})$ stands for the Hurewicz homomorphism and $w_{1}(M)$ for the first Stiefel-Whitney class regarded as a homomorphism via the homomorphism of the universal coefficient Theorem

$$
H^{1}\left(M ; \mathbb{Z}_{2}\right) \longrightarrow \operatorname{Hom}\left(H_{1}(M ; \mathbb{Z}), \mathbb{Z}_{2}\right)
$$

and $C_{2}$ is identified with $\mathbb{Z}_{2}$.

Remark 2.1. Let $M^{m}$ is a connected non-orientable manifold. 
1. If the 1-skeleton $M^{1}$ of a certain $C W$-decomposition of $M$ is a strong deformation retract of some of its open neighbourhood $U$, then the complement $M \backslash U$ cannot be the vanishing set of any top form on $M$, as the group homomorphism

$$
\pi_{1}\left(i_{M \backslash U}\right): \pi_{1}(M \backslash U) \longrightarrow \pi_{1}(M)
$$

is onto [11, p. 39].

2. The $m-2$ and lower dimensional submanifolds of $M^{m}$ cannot be the vanishing sets of any top forms on $M$, as the group homomorphism

$$
\pi_{1}\left(i_{M \backslash X}\right): \pi_{1}(M \backslash X) \longrightarrow \pi_{1}(M)
$$

is an isomorphism for $m \geq 3$ and an epimorphism for $m=2$, whenever $X$ is such a submanifold of $M$. In particular the discrete subsets of $M$ cannot be the vanishing sets of any top forms on $M[16$, Proposition 2.3]. By using the same type of arguments one can actually show that no countable subset of $M$ can be the vanishing sets of any top form on $M$. In other words the vanishing set of every top form on $M$ is uncountable. In fact the zero dimensional subsets of $M$ cannot be the vanishing sets of any top forms on $M$, as we shall see in the Theorem 2.1 and Corollary 2.2.

Theorem 2.1. If $M^{m}$ is a smooth connected manifold $(m \geq 2)$ and $A \subseteq M$ is a closed zero dimensional set, then $M \backslash A$ is also connected and the group homomorphism

$$
\pi_{1}(i): \pi_{1}(M \backslash A) \longrightarrow \pi_{1}(M),
$$

induced by the inclusion $i: M \backslash A \hookrightarrow M$, is onto, i.e. $\pi_{1}(M, M \backslash A)=0$.

Corollary 2.2. If $M^{m}$ is a non-orientable manifold, then $\operatorname{dim} V(\omega) \geq 1$ for every differentiable form $\omega \in \Omega^{m}(M)$.

Proof. Assume that $\operatorname{dim} V(\omega)=0$ for some differentiable form $\omega \in \Omega^{m}(M)$. According to Theorem 2.1, the complement $M \backslash V(\theta)$ of the vanishing set is also connected and the group homomorphism

$$
\pi_{1}(i): \pi_{1}(M \backslash V(\theta)) \longrightarrow \pi_{1}(M)
$$

is onto. The non-orientability of $M$ shows that the orientation character $w_{M}$ is onto. Consequently the orientaion character $\omega_{M \backslash V(\theta)}=w_{M} \circ \pi_{1}\left(i_{M \backslash V(\theta)}\right)$, of $M \backslash V(\theta)$, is also onto, due to Theorem 2.1.

On the other hand the restriction $\left.\theta\right|_{M \backslash V(\theta)}$ is a nowhere vanishing top form of $M \backslash V(\theta)$, which shows that $M \backslash V(\theta)$ is an orientable open submanifold of $M$. In other words, the orientation character $\omega_{M \backslash V(\theta)}=w_{M} \circ \pi_{1}\left(i_{M \backslash V(\theta)}\right)$ is trivial, which implies that either the orientation character $w_{M}$ is not onto or the induced group homomorphism $\pi_{1}\left(i_{M \backslash V(\theta)}\right): \pi_{1}(M \backslash V(\theta)) \longrightarrow \pi_{1}(M)$ is not onto, which is absurd.

In the compact non-orientable case we can provide, by using some different type of arguments, a much larger lower bound for the vanishing sets of all top forms.

Theorem 2.3. If $M^{m}$ is a compact connected non-orientable manifold, then $\operatorname{dim} V(\omega) \geq m-1$ for every differentiable form $\omega \in \Omega^{m}(M)$. 
Remark 2.2. The estimate, provided by Corollary 2.2 is sometimes sharp. Indeed, by removing a suitable circle out of Klein bottle we obtain a cylinder, which is orientable. Also by removing a suitable copy of the $(2 n-1)$-dimensional real projective space, out of the $2 n$-dimensional real projective space we obtain a $2 n$-disc, which is also orientable. In both casses the removed submanifolds have, due to Corollary 2.2 and Theorem 2.3, the smallest possible dimension in order to get orientability on their complements.

\section{Preliminary results}

\subsection{Vanishing sets of differentiable forms}

If $\omega$ is a $k$-differential on $M$, recall that the vanishing set $V(\omega)$ of $\omega$ is the collection of points $z \in U$ at which $\omega$ vanishes, i.e.

$$
V(\omega):=\left\{z \in M: \omega_{z}\left(v_{1}, \ldots, v_{k}\right)=0 \text { for all } v_{i} \in T_{z}(M)\right\} .
$$

We shall only use in this paper the vanishing sets of the top differential forms of $M$.

In this subsection we investigate the size of critical sets of maps between two manifolds with the same dimension via the vanishing set of the pull-back form of a volume form on the target manifold.

Remark 3.1. If $f: M^{n} \rightarrow N^{n}$ is a local diffeomorphism and $\theta \in \Omega^{k}(N)$, then $V\left(f^{*} \theta\right)=$ $f^{-1}(V(\theta))$. If $f$ is additionally surjective, then this equality can be rewritten as $f\left(V\left(f^{*} \theta\right)\right)=V(\theta)$, which shows, by means of Hodel [12],

$$
\operatorname{dim}\left(V\left(f^{*} \theta\right)\right)=\operatorname{dim} V(\theta)
$$

whenever $V\left(f^{*} \theta\right)$ is compact.

Theorem 3.1. ([17]) If $M^{m}, N^{n}, m \geq n$ are differential manifolds with $N$ orientable and $f: M \longrightarrow N$ is a differential map, then $C(f)=V\left(f^{*} \operatorname{vol}_{N}\right)$, where $\operatorname{vol}_{N}$ is a volume form on $N$.

Corollary 3.2. Let $M^{n}, N^{n}$ be differential manifolds. If $N$ is orientable and $M$ is non-orientable then $\operatorname{dim} C(f) \geq 1$ for every differentiable function $f: M \rightarrow N$.

Proof. Let $\operatorname{vol}_{N}$ be a volume form on $N$. Combining Theorem 3.1 with Corollary 2.2 we deduce that $\operatorname{dim} C(f)=\operatorname{dim} V\left(f^{*} \operatorname{vol}_{N}\right) \geq 1$.

In addition to the usefulness of the vanishing sets of differentiable forms in evaluating the size of the critical sets, they are also useful in evaluating the size of the tangency sets [4].

\subsection{Zero dimensional subsets of manifolds}

Lemma 3.3. If $C$ is a closed subset of a smooth manifold $M^{n}$, then there exists a smooth nonnegative function $f: M \longrightarrow \mathbb{R}$ such that $f^{-1}(0)=C$. 
Proof. We first consider an embedding $j: M \hookrightarrow \mathbb{R}^{2 n+1}$, whose existence is ensured by Whitney's embedding theorem.

If $K \subseteq \mathbb{R}^{2 n+1}$ is a closed subset such that $j(C)=K \cap j(M)$, i.e. $j^{-1}(K)=C$, then the required function is $f=g \circ j$, where $g: \mathbb{R}^{2 n+1} \longrightarrow \mathbb{R}$ is a smooth positive function such that $g^{-1}(0)=K$, whose existence is ensured by the Whitney theorem ([18, Théorème 1, p. 17]).

Proposition 3.4. If $A$ is a closed zero dimensional subset of a smooth manifold $M^{n}$, then for each $x \in M$ and every neighbourhood $U$ of $x$, there exists an open neighbourhood $V$ of $x$ such that $V \subseteq U, \partial V \cap A=\emptyset$ and $\partial V$ is smooth.

Proof. If $x \notin A$, then the existence of $V$ is immediate. Assume now that $a \in A$ and consider an open and relatively compact neighbourhood $V^{\prime}$ of $a$ such that $V^{\prime} \subseteq U$ and $\partial V^{\prime} \cap A=\emptyset$. We may assume that $V^{\prime}$ is actually connected, as otherwise we reduce $V^{\prime}$ to its connected component containing $a$. If $\varphi: M \longrightarrow \mathbb{R}$ is a smooth nonnegative function such that $\varphi^{-1}(0)=A$, whose existence is ensured by Lemma 3.3, observe that $m:=\min \left\{\varphi(x) \mid x \in \partial V^{\prime}\right\}>0$, since the compact set $A \cap c l\left(V^{\prime}\right)=A \cap V^{\prime}$ has no common points with the compact boundary $\partial V^{\prime}$. If $y \in(0, m)$ is a regular value of $\left.\varphi\right|_{V^{\prime}}: V^{\prime} \rightarrow \mathbb{R}$, then $\left(\left.\varphi\right|_{V^{\prime}}\right)^{-1}(y)$ is a compact hypersurface in $V^{\prime}$, as $\left(\left.\varphi\right|_{V^{\prime}}\right)^{-1}(y)=$ $\varphi^{-1}(y) \cap \operatorname{cl}\left(V^{\prime}\right)$. Indeed, the inclusion $\left(\left.\varphi\right|_{V^{\prime}}\right)^{-1}(y) \subseteq \varphi^{-1}(y) \cap \operatorname{cl}\left(V^{\prime}\right)$ is obvious. If $x \in \varphi^{-1}(y) \cap \operatorname{cl}\left(V^{\prime}\right)$, then $\varphi(x)=y$ and $x \in \operatorname{cl}\left(V^{\prime}\right)=V^{\prime} \cup \partial V^{\prime}$. But since $y>0$, it follows that $x \notin \partial V^{\prime}$, which shows that $x \in V^{\prime}$ and $x \in\left(\left.\varphi\right|_{V^{\prime}}\right)^{-1}(y)$ as well. Because $y<m$, it follows that $\left(\left.\varphi\right|_{V^{\prime}}\right)^{-1}(y) \cap A=\emptyset$.

Finally, we consider a regular value $y \in(0, m)$ of $\left.\varphi\right|_{V^{\prime}}: V^{\prime} \rightarrow \mathbb{R}$ and observe that the inverse image $\left(\left.\varphi\right|_{V^{\prime}}\right)^{-1}(-\infty, y) \subseteq V^{\prime}$ is an open neighbourhood of $A$ and

$$
\partial\left[\left(\left.\varphi\right|_{V^{\prime}}\right)^{-1}(-\infty, y)\right]=\left(\left.\varphi\right|_{V^{\prime}}\right)^{-1}(y)
$$

which shows that $\partial\left[\left(\left.\varphi\right|_{V^{\prime}}\right)^{-1}(-\infty, y)\right] \cap A=\emptyset$. If $V$ is the connected component of the inverse image $\left(\left.\varphi\right|_{V^{\prime}}\right)^{-1}(-\infty, y)$ containing $a$, then its boundary is a collection of connected components of $\left(\left.\varphi\right|_{V^{\prime}}\right)^{-1}(y)$ and therefore $\partial V \cap A=\emptyset$.

Remark 3.2. If $A$ is a closed zero dimensional subset of a smooth surface $\Sigma$, then for each $x \in \Sigma$ and every neighbourhood $U$ of $x$, there exists an open disk $D$ such that $x \in D \subseteq U, \partial D \cap A=\emptyset$ and $\partial D$ is a smooth circle. Indeed, we consider, via Proposition 3.4, a local chart $(W, \psi)$ of $\Sigma$ at $x$ as well as a connected neighbourhood $V$ of $x$ with smooth boundary such that $x \in V, \operatorname{cl}(V) \subseteq W \subseteq U, \psi(W)=D^{2}$ and $\partial V \cap A=\emptyset$. Note that the boundary of $\psi(V)$ is a union of pairwise disjoint circles, as the circle is the only compact boundaryless one dimensional manifold. One of these circles, say $C$, is the boundary of the unbounded component of $\mathbb{R}^{2} \backslash \psi(V)$. The bounded component of $\mathbb{R}^{2} \backslash C$ is completely contained in $D^{2}$, contains $\psi(V)$ and we may choose its inverse image through $\psi$ to play the role of $D$.

\subsection{Deformations of punctured manifolds}

Since the deformations of the punctured Euclidean space and the punctured manifolds [16] will be repeatedly used in what follows, we shall review them shortly. 
For $r>0$ and $n \in \mathbb{N}^{*}$ denote by $D_{r}^{n}$ and $S_{r}^{n-1}$ the open disk and the sphere respectively, both of them having the center at the origin of the space $\mathbb{R}^{n}$ and radius $r . D_{1}^{n}$ and $S_{1}^{n-1}$ will be simply denoted by $D^{n}$ and $S^{n-1}$ respectively. For $x \in D^{n}$, consider the map $h_{x}: \mathbb{R}^{n} \backslash\{x\} \longrightarrow \mathbb{R}^{n} \backslash\{x\}$ defined to be the identity outside the open disc $D^{n}$ and $\left.h_{x}(y)=S^{n-1} \cap\right] x y$ for every $y \in D^{n} \backslash\{x\}$, where $] x y$ stands for the half line $\{(1-s) x+s y: s>0\}$. In particular $h_{x}(y)=y, \forall y \in S^{n-1}$.

Let $N$ be an $n$-dimensional manifold and $c=(U, \varphi)$ be a local chart of $N$ such that $\operatorname{cl}\left(D^{n}\right) \subseteq \varphi(U)$. Denote by $D_{\varphi}$ and $S_{\varphi}$ the sets $\varphi^{-1}\left(D^{n}\right)$ and $\varphi^{-1}\left(S^{n-1}\right)$ respectively. For $x \in D_{\varphi}$ we define the continuous map $h_{c, x}: N \backslash\{x\} \longrightarrow N \backslash\{x\}$ by

$$
h_{c, x}(y)=\left\{\begin{array}{cl}
y & \text { if } y \in M \backslash D_{\varphi} \\
\varphi^{-1}\left(h_{\varphi(x)}(\varphi(y))\right) & \text { if } y \in U \backslash\{x\} .
\end{array}\right.
$$

Note that $h_{c, x}\left(D_{\varphi} \backslash\{x\}\right)=S_{\varphi}$ and $h_{c, x}(y)=y, \forall y \in S_{\varphi}$.

Remark 3.3. $\quad$ 1. $h_{x}\left(D^{n} \backslash\{x\}\right)=S^{n-1}$ and $h_{x} \simeq_{H_{x}} i d_{\mathbb{R}^{n} \backslash\{x\}}\left(\right.$ rel $\left.\mathbb{R}^{n} \backslash D^{n}\right)$, where

$$
H_{x}: \mathbb{R}^{n} \backslash\{x\} \times[0,1] \rightarrow \mathbb{R}^{n} \backslash\{x\}, H_{x}(y, t)=(1-t) y+t h_{x}(y) .
$$

2. $h_{c, x} \simeq_{H_{c, x}} i d_{M \backslash\{x\}}$, where $H_{c, x}:(M \backslash\{x\}) \times[0,1] \rightarrow M \backslash\{x\}$,

$$
H_{c, x}(y, t)=\left\{\begin{array}{cl}
y & \text { if } y \in M \backslash D_{\varphi} \\
\varphi^{-1}\left(H_{\varphi(x)}(\varphi(y), t)\right) & \text { if } y \in U \backslash\{x\}
\end{array}\right.
$$

If $P$ is a given manifold and $f: P \longrightarrow M$ is a continuous map whose image avoids the point $x$, then $f \simeq h_{c, x} \circ f$ and a homotopy between $f$ and $h_{c, x} \circ f$ is $H_{c, x}(\cdot, t) \circ f$. We shall refere to each $h_{c, x} \circ f$ and $H_{c, x}(\cdot, t) \circ f$ as the punctured deformation of $f$ from $x$ onto $S_{\varphi}$.

\section{The proofs of theorems 2.1 and 2.3}

Proof of Theorem 2.1. Consider a homotopy class of curves in $\pi_{1}(M, M \backslash A)$ represented by a continuous curve $\alpha:[0,1] \longrightarrow M, \alpha(0), \alpha(1) \in M \backslash A$ and deform $\alpha$ $\operatorname{rel}\{0,1\}$ to some differentiable curve $\beta$ with non vanishing tangent vector field. The immersion $\beta$ might actually be chosen to be a geodesic from $\alpha(0)$ to $\alpha(1)$ with respect to some Riemannian metric on $M$ (see e.g. [14, Theorem 1.4.6, p. 24]). Obviously $\operatorname{dim}(A \cap \operatorname{Im}(\beta)) \leq \operatorname{dim}(A)=0$ and $\operatorname{dim} \operatorname{Im}(\beta)=1$.

From this point we continue the proof by induction with respect to the dimension $m$ of the manifold $M$. First assume that $m=2$ and observe that for each $t \in \beta^{-1}(A)$ there exists, via Remark 3.2, a two dimensional disc $D_{t} \subseteq M$ with circular boundary, neighbourhood of $\beta(t)$, such that its circular boundary $C_{t}$ has no common points with $A$. Since $\beta$ is locally an embedding, $D_{t}$ might be chosen inside the domain $U_{t}$ of a coordinate chart $c_{t}=\left(U_{t}, \varphi_{t}\right)$ in such a way that $D_{t}=D_{\varphi_{t}}, C_{t}=S_{\varphi_{t}}$, $\alpha(0)=\beta(0), \alpha(1)=\beta(1) \in M \backslash \operatorname{cl}\left(D_{t}\right), J_{t}:=\beta^{-1}\left(D_{t}\right)$ is an open interval and $\varphi_{t}\left(D_{t} \cap \operatorname{Im}\left(\left.\beta\right|_{J_{t}}\right)\right)=\varphi\left(D_{t}\right) \cap \mathbb{R}$. Since $\left\{D_{t} \mid t \in \beta^{-1}(A)\right\}$ is an open covering of the compact set $\operatorname{Im}(\beta) \cap A$, we may extract a finite open cover, say $D_{t_{1}}, \ldots, D_{t_{s}}$. We may 
assume that $D_{t_{i}} \backslash \operatorname{cl}\left(D_{t_{j}}\right) \neq \emptyset$ whenever $i \neq j$. Since $\operatorname{dim}(\operatorname{Im}(\beta)=1$ it follows that $\operatorname{Im}(\beta)$ cannot fill the open set

$$
D_{t_{i}} \backslash \bigcup_{\substack{j=1 \\ j \neq i}}^{r} \operatorname{cl}\left(D_{t_{j}}\right)
$$

For each $i \in\{1, \ldots, r\}$, consider a point

$$
x_{i} \in D_{t_{i}} \backslash\left(\operatorname{Im}(\beta) \cup \bigcup_{\substack{j=1 \\ j \neq i}}^{r} \operatorname{cl}\left(D_{t_{j}}\right)\right)
$$

and the maps $g_{c_{t_{i}}, x_{i}},: M \backslash\left\{x_{1}, \ldots, x_{s}\right\} \longrightarrow M \backslash\left\{x_{1}, \ldots, x_{s}\right\}$ given by

$$
g_{c_{t_{i}}, x_{i}}(y)=h_{c_{t_{i}, x_{i}}}(y) \text {. }
$$

Note that $\operatorname{id}_{M \backslash\left\{x_{1}, \ldots, x_{s}\right\}} \simeq g_{c_{t_{1}}, x_{1}} \circ \cdots \circ g_{c_{t_{s}}, x_{s}}\left(\right.$ rel $\left.M \backslash\left(D_{t_{1}} \cup \cdots \cup D_{t_{s}}\right)\right)$ as each of the maps $g_{c_{t_{1}}, x_{1}}, \ldots, g_{c_{t_{s}}, x_{s}}$ is homotopic to $\operatorname{id}_{M \backslash\left\{x_{1}, \ldots, x_{s}\right\}}$ relative to $M \backslash\left(D_{t_{1}} \cup \cdots \cup D_{t_{s}}\right)$. Thus

$$
\beta \simeq\left(g_{c_{t_{1}}, x_{1}} \circ \cdots \circ g_{c_{t_{s}, x_{s}}} \circ \beta\right)(\operatorname{rel}\{0,1\})
$$

and $\operatorname{Im}\left(g_{c_{t_{1}}, x_{1}} \circ \cdots \circ g_{c_{t_{s}}, x_{s}} \circ \beta\right) \subseteq M \backslash A$, as

$$
\left(g_{c_{t_{1}}, x_{1}} \circ \cdots \circ g_{c_{t_{s}}, x_{s}}\right)\left(\left(D_{t_{1}} \cup \cdots \cup D_{t_{s}}\right) \backslash\left\{x_{1}, \ldots, x_{s}\right\}\right) \subseteq C_{t_{1}} \cup \cdots \cup C_{t_{s}} \subseteq M \backslash A
$$

and $\beta^{-1}\left(M \backslash\left(D_{t_{1}} \cup \cdots \cup D_{t_{s}}\right)\right) \subseteq \beta^{-1}(M \backslash A)$.

We next assume that the statement holds for $(m-1)$-dimensional manifolds and we shall prove it for the $m$-dimensional manifold $M$. In this respect we consider a partition $0=t_{0}<t_{1}<\cdots<t_{r}=1$ of the interval $[0,1]$ with small enough norm such that:

1. $\beta\left(\left[t_{0}, t_{1}\right]\right) \cap A=\beta\left(\left[t_{r-1}, t_{r}\right]\right) \cap A=\emptyset$ and $\beta\left(t_{1}\right), \ldots, \beta\left(t_{r-1}\right) \in M \backslash A$.

2. there are small enough open discs $D_{1}=D_{\varphi_{1}}, \ldots, D_{r-2}=D_{\varphi_{r-2}}$ with spherical boundaries $S_{1}=S_{\varphi_{1}}, \ldots, S_{r-2}=S_{\varphi_{r-2}}$, for some charts $c_{1}=\left(U_{1}, \varphi_{1}\right), \ldots, c_{r}=$ $\left(U_{r-2}, \varphi_{r-2}\right)$, with the following properties:

(a) $\beta^{-1}\left(D_{i}\right)$ is the open interval $\left(t_{i}, t_{i+1}\right)$ and the restriction $\left(t_{i}, t_{i+1}\right) \longrightarrow D_{i}$, $t \mapsto \beta(t)$ is an embedding, for every $i=\overline{1, r-2}$;

(b) $\operatorname{cl}\left(D_{i}\right) \cap \operatorname{Im}(\beta)=S_{i} \cap \operatorname{Im}(\beta)=\left\{\beta\left(t_{i}\right), \beta\left(t_{i+1}\right)\right\}$ and $D_{i} \cap D_{i+1}=\emptyset$ while $\operatorname{cl}\left(D_{i}\right) \cap \operatorname{cl}\left(D_{i+1}\right)=S_{i} \cap S_{i+1}=\left\{\beta\left(t_{i+1}\right)\right\}$, for every $i=\overline{1, r-3}$.

Note that $\operatorname{Im}(\beta) \cap A \subset D_{1} \cup \cdots \cup D_{r-2}$. For every $i \in\{1, \ldots, r-2\}$, consider $x_{i} \in D_{i} \backslash$ $\operatorname{Im}(\beta)$ and observe that $\left.\left.\beta\right|_{\left[x_{i}, x_{i+1}\right]} \simeq h_{c_{t_{i}}, x_{i}} \circ \beta\right|_{\left[x_{i}, x_{i+1}\right]}\left(\operatorname{rel}\left(\left\{x_{i}, x_{i+1}\right\}\right)\right)$. By applying the inductive hypothesis to the punctured deformation $\left.h_{c_{t_{i}}, x_{i}} \circ \beta\right|_{\left[x_{i}, x_{i+1}\right]}\left(\operatorname{rel}\left(\left\{x_{i}, x_{i+1}\right\}\right)\right)$ of $\left.\beta\right|_{\left[x_{i}, x_{i+1}\right]}$ from $x_{i}$ onto $S_{i}$, whose image is contained in the $(m-1)$-dimensional sphere $S_{i}$, one can conclude that $\left.h_{c_{t_{i}}, x_{i}} \circ \beta\right|_{\left[x_{i}, x_{i+1}\right]}$ is homotopic $\operatorname{rel}\left(\left\{x_{i}, x_{i+1}\right\}\right)$ to some continuous curve $\gamma_{i}:\left[x_{i}, x_{i+1}\right] \longrightarrow S_{i}$ whose image avoids the set $A$, i.e. $\gamma_{i}\left(\left[x_{i}, x_{i+1}\right]\right) \subseteq S_{i} \backslash A$. Thus $h_{c_{t_{i}}, x_{i}} \circ \beta$ is homotopic rel $(\{0,1\})$ to the continuous curve $\gamma:[0,1] \longrightarrow M \backslash A$ defined by $\left.\gamma\right|_{\left[x_{0}, x_{1}\right]}=\left.\beta\right|_{\left[x_{0}, x_{1}\right]},\left.\gamma\right|_{\left[x_{i}, x_{i+1}\right]}=\gamma_{i}$ for $1 \leq i \leq r-2$ and $\left.\gamma\right|_{\left[x_{r-1}, x_{r}\right]}=\left.\beta\right|_{\left[x_{r-1}, x_{r}\right]}$.

Proof of Theorem 2.3. We first observe that every top-form $\omega \in \Omega^{m}(M)$ is exact, as the top de Rham cohomology group $H_{d R}^{m}(M)$ is trivial [15, Th. 15.21, p. 405], i.e. 
$\omega=d \theta$ for some $\theta \in \Omega^{m-1}(M)$. If $p: \tilde{M} \longrightarrow M$ is the orientable double cover, then $p^{*} \omega=p^{*}(d \theta)=d\left(p^{*} \theta\right)$, which shows that $\int_{\tilde{M}} p^{*} \omega=0$ and that $\operatorname{dim}\left(V\left(p^{*} \omega\right)\right) \geq m-1$ therefore. Thus

$$
\operatorname{dim}(V(\omega))=\operatorname{dim}\left(p\left(V\left(p^{*} \omega\right)\right)\right)=\operatorname{dim}\left(V\left(p^{*} \omega\right)\right) \geq m-1,
$$

as $p\left(V\left(p^{*} \omega\right)\right)=V(\omega)$.

Corollary 4.1. Let $M^{n}$, $N^{n}$ be differential manifolds. If $N$ is orientable and $M$ is compact and non-orientable then $\operatorname{dim} C(f) \geq n-1$ for every differentiable function $f: M \rightarrow N$.

Proof. Let $\operatorname{vol}_{N}$ be a volume form on $N$. Combining Theorem 3.1 with Theorem 2.3 we deduce that $\operatorname{dim} C(f)=\operatorname{dim} V\left(f^{*} \operatorname{vol}_{N}\right) \geq n-1$, for every differentiable function $f: M \longrightarrow N$.

A proof of Corollary 4.1 of similar flavor appears in [17, Theorem 2.4.(b)].

Remark 4.1. Corollaries 3.2 and 4.1 rely on the orientability of the regular set $R(f)=M \backslash C(f)$ in the $0=\operatorname{dim}(N)-\operatorname{dim}(M)$ codimension case which is a priori ensured by the nowhere vanishing restricted top form $\left.f^{*} \operatorname{vol}_{N}\right|_{R(f)}$ on $R(f)$. In the lower codimension case $\operatorname{dim}(M)>\operatorname{dim}(N)$, the lack of orientability of the regular set is obvious, even for the orientable option of the target manifold $N$. We stress this by the example of the projection of a product $M=N \times X$ on the first factor, when $N$ is orientable and $X$ is non-orientable. The critical set of this projection is obviously empty, but its regular set is the whole non-orientable product $M=N \times X$.

However, the orientability of the regular set $R(f)$ ensure similar lower bounds even in the lower codimensional context. More precisely, if $N^{n}$ is orientable and $M^{m}$ $(m>n)$ is connected non-orientable and $f: M \longrightarrow N$ is a differentiable function with orientable regular set $R(f)$, then $\operatorname{dim}(C(f)) \geq 1$. The proof of this statement works along the same lines with the one of Corollary 2.2 , the role of the vanishing set $V(\theta)$ is played here by the critical set $C(f)$.

Acknowledgment. The author is grateful to the anonymous referee for his (or her) useful comments, which have helped him to improve the presentation.

\section{References}

[1] Andrica, D., Funar, L., On smooth maps with finitely many critical points, J. London Math. Soc., 69(2004), no. 2, 783-800.

[2] Andrica, D., Funar, L., Addendum: "On smooth maps with finitely many critical points" J. London Math. Soc., 73(2006), no. 1, 231-236.

[3] Andrica, D., Funar, L., Kudryavtseva, E., On the minimal number of critical points of smooth maps between closed manifolds, Russ. J. Math. Phys., 16(2009), no. 3, 363-370.

[4] Balogh, Z.M., Pintea, C., Rohner, H., Size of tangencies to non-involutive distributions, Indiana Univ. Math. J. 60 (2011), 2061-2092.

[5] Church, P.T., Timourian, J.G., Differentiable maps with 0-dimensional critical set, Pacific J. Math., 41(1972), no. 3, 615-630. 
[6] Church, P.T., Timourian, J.G., Maps with 0-dimensional critical set, Pacific J. Math., 57(1975), no. 1, 59-66.

[7] Engelking, R., Dimension Theory, North-Holland, Amsterdam, 1978.

[8] Funar, L., Global classification of isolated singularities in dimensions $(4,3)$ and $(8,5)$, Ann. Scuola Norm. Sup. Pisa Cl. Sci., 10(2011), 819-861.

[9] Funar, L., Pintea, C., Manifolds which admit maps with finitely many critical points into spheres of small dimensions, Michigan Math. J., 67(2018), 585-615.

[10] Funar, L., Pintea, C., Zhang, P., Examples of smooth maps with finitely many critical points in dimensions $(4,3),(8,5)$ and $(16,9)$, Proc. Amer. Math. Soc., 138(2010), no. 1, 355-365.

[11] Hatcher, A., Algebraic Topology, Cambridge University Press, 2002.

[12] Hodel, R.E., Open functions and dimension, Duke Math. J., 30(1963), 46-468.

[13] Hurewicz, W., Wallman, H., Dimension theory, Princeton Mathematical Series, 4, Princeton University Press, Princeton, NJ, 1941.

[14] Jost, J., Riemannian Geometry and Geometric Analysis, (Third Edition), SpringerVerlag, 2002.

[15] Lee, J.M., Introduction to Smooth Manifolds, Springer, 2006.

[16] Pintea, C., Differentiable mappings with an infinite number of critical points, Proc. Amer. Math. Soc., 128(2000), no. 11, 3435-3444.

[17] Pintea, C., Smooth mappings with higher dimensional critical sets, Canad. Math. Bull., 53(2010), no. 3, 542-549.

[18] Postnikov, M., Leçons de Géométrie, Ed. MIR Moscou, 1990.

Cornel Pintea

Babeş-Bolyai University,

Faculty of Mathematics and Computer Sciences,

1, Kogălniceanu Street,

400084 Cluj-Napoca, Romania

e-mail: cpintea@math.ubbcluj.ro 